\title{
SOME DEVELOPMENTS IN THE THEORY OF INEXTENSIBLE NETWORKS*
}

\author{
By \\ A. C. PIPKIN \\ Brown University
}

1. Introduction. Rivlin [1] has formulated a continuum theory of plane deformations of networks formed by inextensible cords, and has obtained general solutions of both displacement and traction boundary-value problems. This work is summarized in a review article by Rivlin [2]. The theory is most directly applicable to large deformations of wide-mesh nets, since the network is idealized as having no elastic resistance to distortion.

Rivlin's theory gives a reasonable approximation to the large-deformation behavior of closely-woven fabrics, but such networks do exhibit elastic resistance to shearing deformations, especially if the fabric is coated with an elastic material. Adkins [3] generalized Rivlin's theory in several ways, and in particular allowed for nonlinear elastic shearing response.

In the present paper we consider a theory that generalizes Rivlin's theory only slightly, by allowing for shear resistance. Unlike Adkin's theory, we restrict attention to initially rectangular networks, so many of Rivlin's results can be used without change.

The main purpose of the present paper is to draw attention to three kinds of singular behavior that can be exhibited by solutions of boundary-value problems in Rivlin's theory: singular fibers, collapsed regions, and folds. Unless the possibility of such singularities is recognized, one might erroneously conclude that some boundary-value problems have no solution.

A secondary purpose is to point out that if the shearing stress is taken to be proportional to the tangent of the shear angle, it is possible to integrate the equilibrium equations explicitly, much as Rivlin did for the case of no shearing stress.

In Secs. 2 to 4 we present Rivlin's kinematic results and, in addition, discuss the kinematic conditions governing collapsed regions and folds. In Sec. 5 we present Rivlin's representation of the stress in terms of a stress potential, as amended by the inclusion of shearing stress, and we briefly discuss the stress-deformation relation for the shearing stress in Sec. 6.

Since we do not expect solutions within the present theory to be physically reasonable when they predict compressive stresses, in Sec. 7 we derive the conditions on the stress components that are necessary if compressive stresses are to be absent.

\footnotetext{
* Received February 14, 1980. The work described in this paper was carried out under a grant from the National Science Foundation, and was performed while the author was at the University of Nottingham on a visit supported by the Science Research Council. He gratefully acknowledges this support. Special thanks are due to Dr. T. G. Rogers, who contributed materially to the present work because of his interest and his helpful discussions.
} 
A collapsed region is a finite area of the original network that maps onto a single curve in the deformed state. This is mathematically possible in a theory with no shear resistance. In Sec. 8 we show that even in this case, the collapse curve must be a straight line if the region is under tension. The stress distribution in a collapsed region is statically indeterminate in the present theory.

Singular fibers are fibers (or cords, in Rivlin's terminology) in which the stress has a Dirac delta singularity, representing a finite force carried by a single fiber. This behavior is familiar from other theories involving inextensible fibers $[4,5]$. This is discussed in Sec. 9, and an example involving singular fibers is given in Sec. 12.

A fold along a fiber can be under stress, but we show in Sec. 10 that folds that cross both families of fibers are equivalent to traction-free boundaries, if no compressive stresses are present.

In Sec. 11 we show how to integrate the equilibrium equations when the shear resistance has a special form, and we use this solution in Sec. 12 to obtain the stress distribution in a simple problem.

A final purpose of the present paper is to lay the groundwork for a discussion of pure traction boundary-value problems. For networks with no shear resistance, Rivlin showed how to solve such problems if collapsed regions and folds are absent. Here we do not discuss this problem, except to give a rather trivial example in Sec. 13 which illustrates the necessity of taking folds into account.

2. Kinematics. We consider a plane sheet or net formed by two intersecting families of fibers. The fibers initially lie parallel to the $X$ and $Y$ directions of a system of Cartesian coordinates. The network is treated as a continuum, so that every line in the $X$ or $Y$ direction is regarded as a fiber. The constant value of $X$ or $Y$ along a fiber is to be used as a Lagrangian coordinate identifying that fiber even after it is deformed.

We consider only plane deformations, in which the particle initially at $(X, Y)$ goes to the place $\mathbf{x}(X, Y)$ with coordinates $x(X, Y)$ and $y(X, Y)$. Because both families of fibers undergo the same deformation, we are assuming that there is no slippage between fibers of the two different families.

The fibers are treated as inextensible; no element of any fiber can change length in any kinematically admissible deformation. This is a reasonable approximation to the behavior of fibers that are under tension, but not for fibers under compression, since compressive stresses are likely to cause out-of-plane buckling. Consequently, if the solution of a given problem shows that some fibers are carrying compressive stresses, we do not expect the solution to be physically reasonable.

In the deformation from $(X, Y)$ to $\mathbf{x}(X, Y)$, a fiber element $d X$ is mapped onto an element $d \mathbf{x}=\mathbf{a} d X$, say, and since the element must still have the length $d X$, the vector a must be a unit vector. The field of vectors $\mathbf{a}$ is the field of unit vectors tangent to the deformed fibers $Y=$ constant. Similarly, an element $d Y$ maps onto $d \mathbf{x}=\mathbf{b} d Y$, where $\mathbf{b}$ is a unit vector. Thus, the deformation satisfies the conditions

$$
\mathbf{x}_{X}=\mathbf{a}, \quad \mathbf{x}_{Y}=\mathbf{b}, \quad \mathbf{a} \cdot \mathbf{a}=\mathbf{b} \cdot \mathbf{b}=1 .
$$

These inextensibility conditions imply that

$$
\mathbf{a}_{Y}=\mathbf{x}_{X Y}=\mathbf{b}_{X} .
$$

Now, since $\mathbf{a} \cdot \mathbf{a}=1$ then $\mathbf{a} \cdot \mathbf{a}_{Y}=0$, so either $\mathbf{a}_{Y}$ is orthogonal to $\mathbf{a}$ or it is zero. 
Similarly, $\mathbf{b}_{X}$ is either orthogonal to $\mathbf{b}$ or zero. If $\mathbf{a}$ and $\mathbf{b}$ are not parallel, then $\mathbf{a}_{Y}$ and $\mathbf{b}_{X}$ are not parallel, so they can be equal only if both are zero. Thus

$$
\mathbf{a}=\mathbf{a}(X) \quad \text { and } \quad \mathbf{b}=\mathbf{b}(Y) .
$$

By using these results in the relation

$$
d \mathbf{x}=\mathbf{a} d X+\mathbf{b} d Y
$$

and integrating, we find that

$$
\mathbf{x}=\mathbf{f}(X)+\mathbf{g}(Y),
$$

where $\mathbf{f}$ and $\mathbf{g}$ are integrals of $\mathbf{a}$ and $\mathbf{b}$, respectively. Thus, all of the fibers in a given family lie along congruent curves when the network is deformed [1].

If $\mathbf{a}= \pm \mathbf{b}$ throughout some region, the argument leading to (2.5) is not valid there. Instead, if $\mathbf{a}=\mathbf{b}$, say, so that $\mathbf{x}_{X}=\mathbf{x}_{Y}$, then

$$
\mathbf{x}=\mathbf{f}(X+Y) .
$$

Thus, the original region in the $X, Y$ plane maps onto a curve. We call the domain in the $X, Y$ plane a collapsed region, and call the curve $\mathbf{x}=\mathbf{f}$ a collapse curve. If $\mathbf{a}=-\mathbf{b}$ in the collapsed region, the collapse curve has the form

$$
\mathbf{x}=\mathbf{f}(X-Y) \text {. }
$$

For networks with no resistance to shearing, any curve can be a collapse curve if there is no tensile stress in any of the fibers in it. However, we show in Sec. 8 that if the collapsed region is under tension, the collapse curve must be a straight line. With a and $\mathbf{b}$ then constant as well as parallel, (2.4) shows that $\mathbf{x}=X \mathbf{a}+Y \mathbf{b}+\mathbf{c}$, where $\mathbf{c}$ is a constant. Then, although the deformation has the form (2.6) or (2.7), it is also of the form (2.5) that is valid for non-collapsed regions. This means that there is no real loss of generality in assuming that the deformation has the form (2.5) everywhere. However, it should be borne in mind that in a region under no stress, the deformation may be highly indeterminate.

3. Kinematic boundary value problems. Pure displacement boundary-value problems are ill-posed in the present theory, because it is not permissible to specify the final position $\mathbf{x}$ of more than one particle on each fiber. It is permissible to specify the deformed shapes of two intersecting fibers, or to specify the final positions of all of the particles along a curve that is not a fiber.

For example, suppose that two edges of a sheet lie along the fibers $X=0$ and $Y=0$. We may specify that the corner $X=Y=0$ remains fixed at the point $\mathbf{x}=\mathbf{0}$, and that the edges $Y=0$ and $X=0$ lie along the curves $\mathbf{x}=\mathbf{f}(s)$ and $\mathbf{x}=\mathbf{g}(s)$, respectively, where $\mathbf{f}(0)=\mathbf{g}(0)=\mathbf{0}$ and $s$ represents arc length measured from the corner. Then the final location of the particle $(X, 0)$ must be $\mathbf{x}=\mathbf{f}(X)$ in order to satisfy the inextensibility condition along the boundary fiber. Assuming that the sheet is not collapsed, the deformation then takes the form $(2.5)$ at all particles $X, Y$ for which the two fibers $X=$ constant and $Y=$ constant both cross the prescribed boundary curves. Although this solution is of the type normally to be expected, there are other solutions in which the network is partly or completely collapsed, and solutions involving folds. We discuss folds in Sec. 4. 
It is possible to specify $\mathbf{x}(X, Y)$ almost arbitrarily along a boundary arc that is not a fiber, and the deformation is then determined almost uniquely in the triangular region formed by that arc and two of the fibers through its ends [1]. The restriction that must be observed is that the final positions $\mathbf{x}_{A}$ and $\mathbf{x}_{B}$ of two particles cannot be further apart than the total length of the fibers connecting them. If one particle can be reached from the other by traveling along only two fibers, this restriction means that

$$
\left|\mathbf{x}\left(X_{A}, Y_{A}\right)-\mathbf{x}\left(X_{B}, Y_{B}\right)\right| \leq\left|X_{A}-X_{B}\right|+\left|Y_{A}-Y_{B}\right| .
$$

Let $X=X(\theta)$ and $Y=Y(\theta)$ be the undeformed boundary of the sheet, and for illustration let us consider an arc $\theta_{1} \leq \theta \leq \theta_{2}$ on which $X$ and $Y$ increase monotonically from $\left(X_{1}, Y_{1}\right)$ to $\left(X_{2}, Y_{2}\right)$. We specify that the particle numbered $\theta$ goes to the place $\mathbf{x}=\mathbf{f}(\theta)$. The function $\mathbf{f}$ must be such that for all pairs of values of $\theta,(3.1)$ is satisfied.

Our aim is to determine $\mathbf{a}$ and $\mathbf{b}$ along the boundary. When this has been done, the deformation in the region $X_{1} \leq X \leq X_{2}, Y_{1} \leq Y \leq Y_{2}$, can be determined in the form (2.5) by integration.

Let $\mathbf{t}(\theta)$ be the unit vector tangential to the deformed boundary and let $s(\theta)$ be arc length measured along it. These are determined from $\mathbf{f}(\theta)$ by using the relation $\mathbf{t} d s=d \mathbf{f}$. Then with (2.4), along the boundary we have

$$
\mathbf{t} d s=\mathbf{a} d X+\mathbf{b} d Y .
$$

From this it follows after a little algebra that

$$
\mathbf{a} \cdot \mathbf{t}=\left(d s^{2}+d X^{2}-d Y^{2}\right) / 2 d s d X
$$

and

$$
\mathbf{b} \cdot \mathbf{t}=\left(d s^{2}+d Y^{2}-d X^{2}\right) / 2 d s d Y .
$$

Thus, the tangential components of $\mathbf{a}$ and $\mathbf{b}$ are determined uniquely by the boundary curve.

Let $\mathbf{k}$ be a unit vector perpendicular to the sheet, forming a right-handed triad with the unit vectors $\mathbf{i}$ and $\mathbf{j}$ along the initial $X$ and $Y$ directions. Then $\mathbf{k} \times \mathbf{t}$ is a unit vector normal to the deformed boundary. If we express $\mathbf{a}$ and $\mathbf{b}$ in terms of components in the directions $\mathbf{t}$ and $\mathbf{k} \times \mathbf{t}$, we may write

$$
\mathbf{a}=(\mathbf{a} \cdot \mathbf{t}) \mathbf{t}-\mathbf{k} \times \mathbf{t}\left[1-(\mathbf{a} \cdot \mathbf{t})^{2}\right]^{1 / 2}
$$

and

$$
\mathbf{b}=(\mathbf{b} \cdot \mathbf{t}) \mathbf{t}+\mathbf{k} \times \mathbf{t}\left[1-(\mathbf{b} \cdot \mathbf{t})^{2}\right]^{1 / 2} .
$$

The signs of the normal components have been chosen so that (3.5) and (3.6) satisfy (3.2) and $\mathbf{a}, \mathbf{b}$, and $\mathbf{k}$ form a right-handed system, provided that $\mathbf{a} \cdot \mathbf{t}$ and $\mathbf{b} \cdot \mathbf{t}$ have the values given by (3.3) and (3.4). These expressions give a and $\mathbf{b}$ parametrically in terms of $\theta$. These are converted into functions of $X$ and $Y$ respectively by inverting $X=X(\theta)$ and $Y=Y(\theta)$.

If the signs of the coefficients of $\mathbf{k} \times \mathbf{t}$ in (3.5) and (3.6) are both changed, the resulting expressions still satisfy (3.2). This gives a solution in which $\mathbf{a}, \mathbf{b}$, and $\mathbf{k}$ form a left-handed system. The meaning of this second solution is that the sheet has turned over, so that material initially on the left of the boundary curve is now on its right. 
The restriction (3.1) enters into the preceding analysis in the form

$$
|d s| \leq|d X|+|d Y| \text {. }
$$

This restriction assures that the expressions (3.3) and (3.4) for $\mathbf{a} \cdot \mathbf{t}$ and $\mathbf{b} \cdot \mathbf{t}$ are no larger than unity in magnitude, which is necessary in order for the solution to be real-valued.

When (3.7) is satisfied as an equality at each point, the boundary is stretched out to its greatest possible length. In this case $\mathbf{a}, \mathbf{b}$, and $\mathbf{t}$ are all equal, for an arc oriented as in the present example. Then with $\mathbf{a}=\mathbf{b}$ on the boundary, it is possible for the region adjacent to the boundary to be a collapsed region in which the deformation has the form (2.6) rather than (2.4). Conditions on the remainder of the boundary must then be used in order to determine which deformation is correct.

4. Folds. Although we have assumed that the deformed sheet occupies a plane region, the motion from the initial to the deformed state may involve an out-of-plane motion in which the sheet turns over or folds back on itself. In terms of the quantity

$$
J=\mathbf{k} \cdot \mathbf{a} \times \mathbf{b},
$$

a region in which $J$ is negative is a region that has turned over, while $J$ is positive in regions that still have their original orientations.

In deriving the expression (2.5) for the deformation we implicitly assumed that $\mathbf{a}$ and b are smooth. However, the result obviously still satisfies the inextensibility condition even if $\mathbf{a}(X)$ or $\mathbf{b}(Y)$ is discontinuous. If $\mathbf{a}(X)$ is discontinuous across a fiber $X$, we call the discontinuity a kink in the a-lines if the sign of $J$ does not change. But if the discontinuity causes a change in the sign of $J$, the sheet has folded back on itself and has a crease along the fiber $X=$ constant. Similarly, a discontinuity in $\mathbf{b}(Y)$ across a fiber $Y=$ constant represents either a kink or a fold, depending on whether or not $J$ has the same sign on both sides.

The fields $\mathbf{a}$ and $\mathbf{b}$ may also be discontinuous across a curve $C$ that is not a fiber itself, but in that case the functions $\mathbf{a}_{+}(X)$ and $\mathbf{a}_{-}(X)$ on the two sides of $C$ are not identical, and the deformation can have the form (2.5) only piecewise, with functions $\mathbf{f}$ and $\mathbf{g}$ on one side of $C$ that are different from the functions $\mathbf{f}$ and $\mathbf{g}$ on the other side.

If the discontinuity line $C$ is not a fiber, then the discontinuity is necessarily a fold, not a kink. For, as we shall explain, the components of $\mathbf{a}$ and $\mathbf{b}$ tangential to the discontinuity must be continuous across it, but their components normal to the discontinuity line must change sign, while keeping the same magnitudes. It is easy to verify that this implies that $J$ changes sign.

To prove the preceding assertions, we need only consider the derivation in Sec. 3 in which we showed how to find the values of $\mathbf{a}$ and $\mathbf{b}$ along a specified boundary curve. Let us now consider this curve to be a discontinuity rather than a boundary. We found that the components of $\mathbf{a}$ and $\mathbf{b}$ tangential to the curve are uniquely determined, and thus in particular have the same values on both sides of the curve. We also found that the normal components are determined up to a common change of sign of both normal components. Thus, the only possible discontinuity is one in which the components of a and $\mathbf{b}$ normal to the discontinuity line both change sign, so that the sheet folds back on itself.

Kinks and folds in collapsed regions are certainly possible, particularly if there is no stress in the collapsed region, but we will not pursue this. For, as explained in Sec. 2, the 
deformation has the form (2.5) when the region is under stress, and thus the preceding analysis is applicable, while if the region is under no stress the deformation can be highly arbitrary.

5. The stress potential. We consider equilibrium under no body forces and no forces on the flat surface of the sheet. Forces are applied only on the edges of the sheet, and are parallel to it.

Consider two curves in the deformed sheet, both running from a reference particle such as $(0,0)$ to the particle $(X, Y)$. The total force on the region enclosed by the two curves must be zero for equilibrium. Let $\mathbf{F}_{1}$ be the force exerted across one of the curves, by the material on the right-hand side, on the material on the left of the curve. (In speaking of the right and left sides of the curve we are referring to the regions occupied by the material before the deformation.) With $\mathbf{F}_{2}$ the similar force for the second curve, the total force on the enclosed region is $\pm\left(\mathbf{F}_{1}-\mathbf{F}_{2}\right)$, so for equilibrium the two forces must have a common value $\mathbf{F}(X, Y)$ that depends only on their endpoints. Then the force exerted across any directed arc is the difference between the values of $\mathbf{F}(X, Y)$ at its ends, or zero for a closed curve. The force across an infinitesimal element $(d X, d Y)$ is $d \mathbf{F}$.

The moment on an arc from $(0,0)$ to $(X, Y)$ is

$$
M \mathbf{k}=\int_{\mathbf{0}}^{\mathbf{x}} \mathbf{x}(X, Y) \times d \mathbf{F}(X, Y),
$$

or, after integration by parts and use of (2.4),

$$
M \mathbf{k}=\mathbf{x}(X, Y) \times \mathbf{F}(X, Y)+\int_{\mathbf{0}}^{\mathbf{x}} \mathbf{F} \times(\mathbf{a} d X+\mathbf{b} d Y) .
$$

For rotational equilibrium $M$, like $\mathbf{F}$, must be path-independent. This requires the integrand in (5.2) to be a perfect differential, whence, with (2.2),

$$
\mathbf{F}_{Y} \times \mathbf{a}=\mathbf{F}_{X} \times \mathbf{b} .
$$

The force across an element of length $d Y$ in the $\mathbf{b}$ direction is $\mathbf{F}_{Y} d Y$. Thus $\mathbf{F}_{Y}$ plays the role of a stress vector. If we resolve it into components in the $\mathbf{a}$ and $\mathbf{b}$ directions in the form

$$
\mathbf{F}_{Y}=T_{a} \mathbf{a}+S \mathbf{b},
$$

then $T_{a}$ represents a tension in the a-fibers, and $S$ is a shearing stress. Similarly,

$$
\mathbf{F}_{X}=-T_{b} \mathbf{b}-S \mathbf{a} .
$$

This is the force per unit length on an element in the a direction. For a directed arc of this kind, the vector - b points toward the material that was initially on the right, so $T_{b}$ is positive if there is a positive tension in the b-lines. The coefficient $S$ in (5.5) must be the same as that in (5.4) in order to satisfy the rotational equilibrium condition (5.3), unless $\mathbf{a}= \pm \mathbf{b}$, a case that we now consider.

In a collapsed region where $\mathbf{a}$ and $\mathbf{b}$ are parallel, the representations (5.4) and (5.5) are not merely a matter of notation since $\mathbf{a}$ and $\mathbf{b}$ do not form a basis. If we accept these relations as remaining valid in a collapsed region, they imply that the forces transmitted by the collapse line are parallel to the line. Since this is physically reasonable, we accept 
it as a constitutive assumption. In that case the rotational equilibrium condition is satisfied identically, whether or not the two coefficients $S$ in (5.4) and (5.5) are equal, so taking them to be equal is another constitutive assumption.

The tensions in individual fibers in the physical network can be calculated approximately by using the results of the continuum theory. Let $n$ be the number of fibers that are parallel to the $X$ direction, per unit of length in the $Y$ direction, when the sheet is undeformed. Then after the deformation, $n$ is the number of a-fibers that cross a $b$-fiber, per unit of length of the $\mathbf{b}$-fiber. Since $T_{a}$ is the force in the a direction, per unit of length of the $\mathbf{b}$-fiber, then $T_{a} / n$ is the tension in each a-fiber. form

The equation of equilibrium, found by eliminating $\mathbf{F}$ from (5.4) and (5.5), has the

$$
\left(T_{a} \mathbf{a}+S \mathbf{b}\right)_{X}+\left(T_{b} \mathbf{b}+S \mathbf{a}\right)_{Y}=\mathbf{0} .
$$

However, it is usually more convenient to determine the stress potential $\mathbf{F}$ directly, and then determine $T_{a}, T_{b}$, and $S$ from (5.4) and (5.5). Formal expressions for these components are simplified by introducing the auxiliary vectors

$$
\mathbf{A}=\mathbf{b} \times \mathbf{k} \quad \text { and } \quad \mathbf{B}=\mathbf{k} \times \mathbf{a} .
$$

We note that

$$
\mathbf{A} \cdot \mathbf{b}=\mathbf{B} \cdot \mathbf{a}=0 \quad \text { and } \quad \mathbf{A} \cdot \mathbf{a}=\mathbf{B} \cdot \mathbf{b}=J,
$$

where $J$ is defined in (4.1). Then, from (5.4) and (5.5),

$$
T_{a}=J^{-1} \mathbf{A} \cdot \mathbf{F}_{Y}, \quad T_{b}=-J^{-1} \mathbf{B} \cdot \mathbf{F}_{X},
$$

and

$$
S=J^{-1} \mathbf{B} \cdot \mathbf{F}_{Y}=-J^{-1} \mathbf{A} \cdot \mathbf{F}_{X} .
$$

6. Stress-deformation relations. The fiber tensions $T_{a}$ and $T_{b}$ do no work in any deformation satisfying the constraint of fiber inextensibility, so they are reactions to that constraint. As such, they are not connected to the deformation by a constitutive equation, but are to be determined by solving the equilibrium equations.

The shearing stress $S$ performs an amount of work $S d(\mathbf{a} \cdot \mathbf{b})$ per unit of initial area when the angle between the $\mathbf{a}$ and $\mathbf{b}$ directions is changed, since $\mathbf{a} \cdot \mathbf{b}$ is the sine of the angle of shear. We consider only elastic response, for which there is a strain energy density $W$ per unit of initial area which depends only on the local distortion of the network. Measuring this distortion by $\mathbf{a} \cdot \mathbf{b}$, from the work-energy relation we find that $S=W^{\prime}(\mathbf{a} \cdot \mathbf{b})$.

Most of the results in the remainder of this paper are independent of the form of $S$ as a function of $\mathbf{a} \cdot \mathbf{b}$, but it is convenient and natural to assume that it is an odd function. For the explicit integration of the equilibrium equations in Sec. 11, we use the assumption that $S$ is proportional to the tangent of the angle of shear:

$$
S=G \mathbf{a} \cdot \mathbf{b} /|J| \text {. }
$$

Here $|J|$, defined by (4.1), is the cosine of the shear angle, which is expressed in terms of $\mathbf{a} \cdot \mathbf{b}$ by

$$
|J|=\left[1-(\mathbf{a} \cdot \mathbf{b})^{2}\right]^{1 / 2} .
$$


The strain energy density corresponding to (6.1) has the particularly simple form $W=G(1-|J|)$. Since $|J|$ is the area of the deformed sheet, per unit initial area, the strain energy is proportional to the decrease in area.

In the pure network theory discussed by Rivlin [1], the shearing stress is identically zero. This may be viewed as the special case of (6.1) in which the shear modulus $G$ is zero. Adkins [3] has set up the general theory of networks with non-zero shear resistance.

7. Positive normal stress. Boundary-value problems within the present theory often have more than one solution, but when this occurs, experience indicates that there will be at most one solution with no compressive stresses. Consequently, it is useful to try to rule out solutions with compressive stresses in advance. To do this, we use the restrictions

$$
T_{a} \geq 0, \quad T_{b} \geq 0, \quad T_{a} T_{b} \geq S^{2} .
$$

As we shall show, these conditions are necessary and sufficient to ensure that the stress state at a point involves no negative normal stress on any element through that point.

It is possible to devise boundary conditions under which there is no solution at all if compressive stresses are not allowed, so we view the restrictions (7.1) as guidelines rather than strict requirements.

To derive the conditions (7.1), consider the force $d \mathbf{F}$ acting across an element $(d X, d Y)$ :

$$
d \mathbf{F}=\left(T_{a} \mathbf{a}+S \mathbf{b}\right) d Y-\left(T_{b} \mathbf{b}+S \mathbf{a}\right) d X .
$$

This is the force exerted by the material that was originally on the right of the directed arc, on the material on its left. A vector normal to the deformed arc $d \mathbf{x}$ and directed toward the material initially on the right is given by

$$
\mathbf{n} d s=d \mathbf{x} \times \mathbf{k} \operatorname{sgn}(J) \text {, }
$$

where $\operatorname{sgn}(J)$ is +1 or -1 depending on whether or not the sheet has turned over. Then the condition that the normal component of stress on the element is non-negative is $\mathbf{n} \cdot d \mathbf{F} \geq 0$. By using the expressing (2.4) for $d \mathbf{x}$ and the definition (4.1) of $J$ in this condition, we obtain

$$
Q=T_{a} d Y^{2}-2 S d X d Y+T_{b} d X^{2} \geq 0 .
$$

The conditions (7.1) are the standard conditions necessary and sufficient to ensure that the quadratic form $Q$ is non-negative definite.

8. Collapsed regions. We now show that, under the conditions (7.1), in a collapsed region either the stress is zero or the collapse curve is a straight line.

With the particular constitutive equation (6.1) for $S, S$ is infinite in collapsed regions, and this would lead to an infinite total force transmitted by the region. Consequently, collapsed regions do not occur when (6.1) is used. For the present demonstration we suppose that $S$ remains finite when $\mathbf{a}= \pm \mathbf{b}$. Assuming that $S$ has the same sign as $\mathbf{a} \cdot \mathbf{b}$, then $\mathbf{b} S$ is equal to $|S| \mathbf{a}$ in either case.

From (2.6) and (2.7), when $\mathbf{b}= \pm \mathbf{a}$ then $\mathbf{a}=\mathbf{a}(X \pm Y)$. Then the stress equation of equilibrium (5.6) reduces to the form

$$
C \mathbf{a}+\left(T_{a}+T_{b}+2|S|\right) \mathbf{a}^{\prime}=\mathbf{0},
$$

where $\mathbf{a}^{\prime}$ is the derivative of $\mathbf{a}$ with respect to its argument, and the expression for $C$, the coefficient of $\mathbf{a}$, is not needed. 
If $\mathbf{a}^{\prime}$ is not zero it is perpendicular to $\mathbf{a}$, so (8.1) yields

$$
T_{a}+T_{b}+2|S|=0 \text {. }
$$

This cannot be satisfied with positive fiber tensions, and with $T_{a}=T_{b}=0$ it can be satisfied only if $S=0$ as well. Thus collapse curves with $\mathbf{a}^{\prime} \neq \mathbf{0}$ can occur only in materials for which $S=0$ at collapse, and then only when there is no stress in the collapsed region.

If $\mathbf{a}^{\prime}=\mathbf{0}$, so that $\mathbf{a}$ is a constant, the collapse curve is a straight line. In such cases the stress distribution in the collapsed region is statically indeterminate, but subject to loose restrictions concerning the total force transmitted by the region. For example, if $\mathbf{a}=\mathbf{b}=\mathbf{u}$, where $\mathbf{u}$ is a constant unit vector, (5.4) and (5.5) imply that $\mathbf{F}$ has the form $\mathbf{u} F(X, Y)+$ const., and then that

$$
T_{a}=F_{Y_{Y}}-S \quad \text { and } \quad T_{b}=-F_{X_{X}}-S .
$$

The scalar function $F$ must be such that $F$ has the correct boundary values at the boundary of the collapsed region, and this may put some restriction on the shape of the region, but $F$ is otherwise arbitrary since there is no further static or kinematic requirement to be satisfied.

9. Singular fibers. Because the fiber tensions $T_{a}$ and $T_{b}$ are not related to the deformation by a stress-strain relation, it is permissible for these tensions to be infinite. However, if $T_{a}$, say, were infinite over a finite $Y$-interval, the resultant force would be infinite, and this situation does not arise. But solutions of boundary-value problems often do involve Dirac delta singularities in $T_{a}$ and $T_{b}$. A term in $T_{a}$ of the form $F(X) \delta\left(Y-Y_{0}\right)$ means that the fiber $Y=Y_{0}$ carries a finite load $F(X)$. Similarly, $T_{b}$ can have singularities of the form $F(Y) \delta\left(X-X_{0}\right)$, representing a finite force carried by the fiber $X=X_{0}$. We call such fibers singular fibers.

From (5.4) we see that if $T_{a}$ has a term of the form mentioned, then the stress potential $\mathbf{F}$ is discontinuous across the fiber $Y_{0}$, and the difference between its values above and below the singular fiber is $F(X) \mathbf{a}(X)$, parallel to the fiber. Similarly, at a fiber $X_{0}$ carrying a load $F(Y)$, the difference between the values of $\mathbf{F}$ on the right and the left is $-\mathbf{b}(Y) F(Y)$, parallel to the fiber and toward the right-hand side of the arc $d X$ that crosses the fiber.

F must be continuous across any curve $C$ that does not lie along a fiber, except in the degenerate case of a collapsed region, where the stress distribution is statically indeterminate. For, on crossing such a curve, the same discontinuity in $\mathbf{F}$ occurs whether the crossing is regarded as being made by an infinitesimal movement in the $X$ direction or the $Y$ direction. But from (5.4) and (5.5), the jump in $\mathbf{F}$ is parallel to $\mathbf{a}$ if the crossing is in the $Y$ direction, and parallel to $\mathbf{b}$ if the crossing is in the $X$ direction. Then if $\mathbf{a}$ and $\mathbf{b}$ are not parallel, the jump must be zero.

10. Equilibrium of folds. If the direction $\mathbf{b}(Y)$ is discontinuous across a fiber $Y=Y_{0}$, that fiber must ordinarily be singular, carrying a finite load. Conversely, because a fiber can carry a finite load, equilibrium conditions do not put any restrictions on permissible jumps in $\mathbf{b}$ (or $\mathbf{a}$, for discontinuities across a fiber $X=X_{0}$ ). In particular, folds can occur.

The directions a and $\mathbf{b}$ may also be discontinuous across a curve that is not a fiber, and we have found in Sec. 4 that the discontinuity is then necessarily a fold. Since the discontinuity curve is not along a fiber, $\mathbf{F}$ must be continuous across it. 
The fold forms a free edge in the deformed sheet, with regions that were originally on opposite sides of the fold now both on the same side, so that normal vectors pointing toward the two regions now point in the same direction. Then for equilibrium, the sum of the normal stresses must be zero. Thus, one of the normal stresses must be compressive, unless both are zero. We consider only the latter case, since we seek solutions with no compressive stresses. (The preceding argument can be carried out algebraically, using the continuity of $\mathbf{F}$ and the conditions on $\mathbf{a}$ and $\mathbf{b}$ at a fold to show that if (7.1) is satisfied, the normal stress on the fold must be zero.)

Let $(d X, d Y)$ be an element of arc along the fold. Then the quadratic form $Q$ defined in (7.4) is zero for this choice of $d X$ and $d Y$. If $Q$ also vanishes for some direction other than the fold direction, then since $Q$ is non-negative definite, it is identically zero and thus $T_{a}, T_{b}$, and $S$ are all zero at the fold. Then $d \mathbf{F}=\mathbf{0}$, and thus $\mathbf{F}$ is constant along the fold.

The conclusion that $\mathbf{F}$ is constant along the fold is still true in the less trivial case in which the fold direction is the only direction for which $Q$ vanishes. In that case, by setting $Q=0$ and solving for $d Y / d X$, we find that the two roots are coincident when

$$
d Y / d X=S / T_{a}=T_{b} / S .
$$

In particular, (7.1c) must be satisfied as an equality.

From Sec. 4 we know that the discontinuities in $\mathbf{a}$ and $\mathbf{b}$ across a fold are such that $\mathbf{a} \cdot \mathbf{b}$ is continuous, and with $S$ a function of $\mathbf{a} \cdot \mathbf{b}, S$ is continuous across the fold. Then since the fold direction given by (10.1) must be the same regardless of the side on which $T_{a}$ and $T_{b}$ evaluated, these tensions must also be continuous across the fold. By using (10.1) in (7.2), we find that $d \mathbf{F}=\mathbf{0}$ along the fold, as asserted.

The conclusion that $\mathbf{F}$ is constant along the fold means that neither of the regions adjacent to the fold exerts any force on it. This result has a simple and important consequence for pure networks, in which $S$ is identically zero: there is no stress in the triangular region bounded by the fold and two fibers $X$ and $Y$ that cross it; $F$ is constant not only along the fold but throughout such a region. To prove this, note that the resultant force on the fiber $Y$ is parallel to $b(Y)$, since this is true pointwise when $S=0$. Similarly, the force on the fiber $X$ is parallel to a $X)$. The sum of these forces must be zero since the boundary at the fold is traction-free. Then, assuming that the triangular region is not a collapsed region, both resultants must be zero, and since this is true for any such triangle, the tensions $T_{a}$ and $T_{b}$ must be zero pointwise. With the same conclusion for similar triangular regions on the other side of the fold, it follows that $\mathbf{F}$ is constant throughout any initially rectangular region with the fold connecting diagonally opposite corners.

11. Integration of the equilibrium equations. Rivlin [1] has shown how to solve (5.4) and (5.5) for $\mathbf{F}$, for the special case of a pure network, and Adkins [3] has obtained similar results for networks with shear resistance. Adkins' general results are difficult to use unless the deformation is known in advance. To obtain results that are explicit enough to be of use in solving traction boundary-value problems, we suppose that the shearing stress $S$ has the special form (6.1). Then from (5.10), with (6.1) we obtain

$$
\mathbf{B}(X) \cdot \mathbf{F}_{Y}(X, Y)=-\mathbf{A}(Y) \cdot \mathbf{F}_{X}(X, Y)=G \mathbf{a}(X) \cdot \mathbf{b}(Y) \operatorname{sgn}(J) \text {. }
$$

We are assuming that the region considered is not a collapsed region. 
We consider a region throughout which $\operatorname{sgn}(J)$ remains constant. This region may be the whole sheet, or it may be bounded partly or entirely by folds. Discontinuities in a and b that do not change the sign of $J$ are permissible. Then integration of (11.1) yields

$$
\mathbf{B} \cdot \mathbf{F}=M(X)+G \mathbf{a}(X) \cdot \mathbf{x}(X, Y) \operatorname{sgn}(J)
$$

and

$$
\mathbf{A} \cdot \mathbf{F}=N(Y)-G \mathbf{b}(Y) \cdot \mathbf{x}(X, Y) \operatorname{sgn}(J)
$$

where $M$ and $N$ are arbitrary. Now, from (5.7) and (5.8),

$$
\mathbf{F}=(\mathbf{F} \cdot \mathbf{A} / J) \mathbf{a}+(\mathbf{F} \cdot \mathbf{B} / J) \mathbf{b}
$$

and

$$
\mathbf{b}(\mathbf{a} \cdot \mathbf{x})-\mathbf{a}(\mathbf{b} \cdot \mathbf{x})=\mathbf{k} \times[\mathbf{A}(\mathbf{a} \cdot \mathbf{x})+\mathbf{B}(\mathbf{b} \cdot \mathbf{x})]=J \mathbf{k} \times \mathbf{x} .
$$

By using (11.2) and (11.3) in (11.4) and making use of (11.5), we obtain

$$
\mathbf{F}=G \operatorname{sgn}(J) \mathbf{k} \times \mathbf{x}(X, Y)+J^{-1}(X, Y)[N(Y) \mathbf{a}(X)+M(X) \mathbf{b}(Y)] .
$$

When the deformation can be determined purely kinematically, as in Sec. 3, all of the quantities in the right-hand member of (11.6) are known except $M$ and $N$. The latter functions are to be determined from traction boundary conditions. This is most easily done when the tractions are specified as forces per unit initial length (dead loading). By integrating these tractions with respect to initial arc length, we find the boundary values of $\mathbf{F}$, up to an arbitrary constant on each connected arc where tractions are specified. Then $M$ and $N$ are determined from the boundary values of $\mathbf{F}$ by using (11.2) and (11.3).

12. Example: sheared sheet. The process of determining the stress field and the manner in which singular fibers arise can be illustrated by a simple example. We consider an initially square sheet with edges at $X=0, L$ and $Y=0, L$. The edge $Y=0$ is fixed, and the edge $X=0$ is sheared over so as to lie along the straight line with tangent vector $\mathbf{b}=s \mathbf{i}+c \mathbf{j}$. Then $\mathbf{b}$ has this same value throughout the sheet, and $\mathbf{a}=\mathbf{i}$. The deformation is $\mathbf{x}=X \mathbf{a}+Y \mathbf{b}$. The auxiliary vectors $\mathbf{A}$ and $\mathbf{B}$ introduced in (5.7) are $\mathbf{A}=c \mathbf{i}-s \mathbf{j}$ and $\mathbf{B}=\mathbf{j}$, and the cosine of the angle of shear is $J=c$.

A dead load $T_{1} \mathbf{i}$ per unit of initial length is applied to the edge $X=L$, and a load $T_{2} \mathbf{j}$ is applied along $Y=L$. Then the boundary values of $F$, denoted $\mathbf{F}_{0}$, can be taken to be

$$
\mathbf{F}_{0}(L, Y)=T_{1} Y \mathbf{i}, \quad \mathbf{F}_{0}(X, L)=T_{1} L \mathbf{i}+T_{2}(L-X) \mathbf{j} .
$$

By using the preceding results in (11.2) and (11.3) we find that

$$
M(X)=T_{2}(L-X)-G(X+s L)
$$

and

$$
N(Y)=T_{1} c Y+G(Y+s L) .
$$

By using these in (11.6) and expressing $\mathbf{i}$ and $\mathbf{j}$ in terms of $\mathbf{a}$ and $\mathbf{b}$, we obtain

$$
\mathbf{F}=\left[T_{1} Y+S(L-X)\right] \mathbf{a}+\left[\left(T_{2} / c\right)(L-X)-S(L-Y)\right] \mathbf{b},
$$

where $S=G s / c$. With (5.4) and (5.5), we find that $T_{a}=T_{1}$ and $T_{b}=T_{2} / c$. 
We note immediately that although the fiber tensions are positive when $T_{1}$ and $T_{2}$ are, the third condition in (7.1) also requires that $T_{1} T_{2} / c \geq(G s / c)^{2}$. If this is not satisfied, compressive stresses are present. Thus if $T_{1}$ and $T_{2}$ are not sufficiently large, we may expect wrinkles to form, which are not described by the present solution.

The boundary values of $\mathbf{F}$ given by (12.4) are not equal to the prescribed boundary values (12.1), even though the latter were used in obtaining (12.4). The discrepancies are

$$
\mathbf{F}_{0}(L, Y)-\mathbf{F}(L, Y)=S(L-Y) \mathbf{b}
$$

and

$$
\mathbf{F}_{0}(X, L)-\mathbf{F}(X, L)=-\left(T_{2}+G\right)(s / c)(L-X) \mathbf{a} .
$$

These results do not indicate that a mistake has been made; they merely show that $\mathbf{F}$ is discontinuous across the boundary. In each case the jump in $F$ is parallel to the fiber across which the discontinuity occurs. The boundary fibers $X=L$ and $Y=L$ are singular, carrying finite loads whose magnitudes are given by (12.5) and (12.6).

It was possible for these discontinuities to arise because $M(X)$ was determined by using (11.2) only along the top boundary, and $N(Y)$ by using (11.3) only along the right-hand boundary. If (11.2) and (11.3) are both used along a boundary that is not a fiber, then both components of $\mathbf{F}$ will be continuous across the boundary.

The forces in the boundary fibers given by (12.5) and (12.6) are positive only if $s$ is negative, so that the sheet is sheared toward the left. When $s$ is positive the boundary fibers are under compression, and we do not expect the present solution to be physically meaningful.

13. Example: folded sheet. For pure networks with $G=0$, Rivlin [1] showed how to solve pure traction boundary-value problems with dead loads. Since the force exerted across a fiber $X$ is parallel to a $(X)$ at every point of that fiber, the direction a must be parallel to the load on the part of the boundary to the right of that fiber; $\mathbf{b}(Y)$ is determined similarly. This method implicitly assumes that no collapsed regions or folds are present.

As a simple example to illustrate the need to consider collapsed regions and folds, let us consider a square sheet like that in Sec. 12, with edges free from traction except for point forces at its corners, equal to $-2 F \mathbf{j}, F(\mathbf{j}-\mathbf{i}), 2 F \mathbf{i}$, and $F(\mathbf{j}-\mathbf{i})$ at $(0,0),(L, 0)$, $(L, L)$ and $(0, L)$, respectively. Then the boundary values of $\mathbf{F}$ can be taken to be

$$
\mathbf{F}_{0}(X, 0)=-F \mathbf{j}, \quad \mathbf{F}_{0}(L, Y)=-F \mathbf{i}, \quad \mathbf{F}_{0}(X, L)=F \mathbf{i}, \quad \mathbf{F}_{0}(0, Y)=F \mathbf{j} .
$$

The load on the part of the boundary to the right of a fiber $X$ is $F(\mathbf{i}+\mathbf{j})$, and this is also the load on the part of the boundary above a fiber $Y$, so both $\mathbf{a}$ and $\mathbf{b}$ are parallel to $\mathbf{i}+\mathbf{j}$ if the deformation has the form (2.5). Then the whole sheet is a collapsed region, and from the results in Sec. 8 we know that $\mathbf{F}$ must be of the form $F(X, Y)(\mathbf{i}+\mathbf{j})+\mathbf{c}$. But it is easy to verify that no choice of $F$ and $\mathbf{c}$ can satisfy the four conditions (13.1), even under the assumption that the boundary fibers are singular.

We have not presented a diagram showing the boundary loads because it would then be all too clear what is happening. A fold will form, running diagonally across the sheet from $(0,0)$ to $(L, L)$. With $G=0, \mathbf{F}$ must be constant not only along the fold but at every interior point, as discussed in Section 10. Let $\mathbf{F}^{*}$ be this constant value. Then the difference between $\mathbf{F}^{*}$ and the boundary values (13.1) give the directions of the boundary 
fibers. The condition that these four directions form a closed loop then gives an equation for the determination of $\mathbf{F}^{*}$. It is easily verified that $\mathbf{F}^{*}=\mathbf{0}$ is a solution. Then the directions of the boundary fibers are $\pm \mathbf{F}_{0}$, the sign depending on which boundary is considered. Thus, from (13.1) we find that

$$
\mathbf{a}(X, 0)=\mathbf{j}, \quad \mathbf{b}(L, Y)=\mathbf{i}, \quad \mathbf{a}(X, L)=\mathbf{i}, \quad \mathbf{b}(0, Y)=\mathbf{j} .
$$

These values are consistent with a deformation $\mathbf{x}=X \mathbf{i}+Y \mathbf{j}$ for $Y \geq X$ (undeformed) and $\mathbf{x}=X \mathbf{j}+Y \mathbf{i}$ for $Y \leq X$, so the latter region is simply folded over. There is a tensile force $F$ in each boundary fiber.

\section{REFERENCES}

[1] R. S. Rivlin, Plane strain of a net formed by inextensible cords, Arch. Rat. Mech. Anal. 4, 951-74 (1955)

[2] R. S. Rivlin, Networks of inextensible cords, in Nonlinear problems of engineering, pp. 51-64, New York, Academic Press (1964)

[3] J. E. Adkins, Finite plane deformation of thin elastic sheets reinforced with inextensible cords, Phil. Trans. Roy. Soc. London 249, 125-50 (1956)

[4] A. C. Pipkin and T. G. Rogers, Plane deformations of incompressible fiber-reinforced materials, J. Appl. Mech. 38, 634-40 (1971)

[5] G. C. Everstine and A. C. Pipkin, Stress channelling in transversely isotropic elastic composites, ZAMP 22, 825-34 (1971) 